\title{
OPINIÃO DAS FAMÍLIAS SOBRE AS ATIVIDADES LÚDICAS DESENVOLVIDAS COM CRIANÇAS NA ESCOLAABORDANDO HÁBITOS SAUDÁVEIS
}

Bárbara Mohr da Silveir Luciara Fabiane Sebold Larissa Evangelista Ferreira Juliana Balbinot Reis Girondi Lúcia Nazarth Amante Juliana Simas Justino

\author{
https://orcid.org/0000-0003-4428-5346 \\ https://orcid.org/0000-0002-5023-9058 \\ https://orcid.org/0000-0002-5407-3761 \\ https://orcid.org/0000-0002-3763-4176 \\ https://orcid.org/0000-0002-5440-2094 \\ https://orcid.org/0000-0001-7413-8270
}

RESUMO: Objetivo conhecer a opinião dos pais acerca de atividades realizadas na escola sobre alimentação saudável e prática de atividades físicas. Metodologia: Estudo descritivo e exploratório de abordagem qualitativa, realizado com 51 famílias e seus respectivos filhos, através de questionário de opinião. Resultados: Nos relatos as família abordaram questões como a mudança de hábitos visualizados em seus filhos após a realização de atividades na escola, assim como deram opiniões sobre o ensino da temática na escola e formas de debater estes temas no ambiente escolar. Conclusão: As atividades de abordagem lúdica possuem significados singular para as crianças no ensino de hábitos saudáveis no ambiente escolar e suas repercussões nas relações familiares podem favorecer a adesão de melhores hábitos de vida.

Descritores: Educação em saúde; Relações Familiares; Enfermagem.

\section{FAMILY VIEWS ON LIVING ACTIVITIES DEVELOPED WITH CHILDREN AT SCHOOLADDRESSING HEALTHY HABITS}

A healthy life can contribute in many ways to the health of individuals. One way to have a healthy life is by eating healthy foods and regular physical activity. The insertion of these habits in daily life from childhood can contribute to a healthier adult life. This study aimed to know the parents' opinion about the activities carried out in the school about healthy eating and physical activity practice. A descriptive and exploratory study of a qualitative approach, carried out with 51 parents and / or guardians and their respective children. In the reports, the parents addressed issues such as the change of habits seen in their children after school activities, as well as opinions about the teaching of the subject at school and ways to discuss these issues in the school environment. Finally, activities of playful approach have singular meanings for children in teaching healthy habits in the school environment.

Descriptores: Health education; Family relationships; Nursing.

\section{OPINIÓN DE LAS FAMILIAS SOBRE LAS ACTIVIDADES LÚDICAS DESARROLLADAS CON NIÑOS EN LA ESCUELA ABORDANDO HÁBITOS SALUDABLES}

Una vida sana puede contribuir de innumerables formas en la salud de los individuos. Una forma de tener una vida saludable es a través del consumo de alimentos saludables y la práctica regular de las actividades físicas. La inserción de estos hábitos en el cotidiano desde la infancia puede contribuir a una vida adulta con más salud. Este estudio tuvo como objetivo conocer la opinión de los padres sobre las actividades realizadas en la escuela sobre alimentación sana y práctica de actividades físicas. Estudio descriptivo y exploratorio de abordaje cualitativo, realizado con 51 padres y / o responsables y sus respectivos hijos. En los relatos los padres abordaron cuestiones como el cambio de hábitos visualizados en sus hijos después de la realización de actividades en la escuela, así como dieron opiniones sobre la enseñanza de la temática en la escuela y formas de debatir estos temas en el ambiente escolar. Por último, las actividades de abordaje lúdico poseen significados singular para los niños en la enseñanza de hábitos saludables en el ambiente escolar.

Descriptores: Educación en salud; Relaciones Familiares; Enfermería.

${ }^{1}$ Imperial Hospital de Caridade,Florianópolis/SC.

2Universidade Federal de Santa Catarina-UFSC/SC

Autor correspondente: Bárbara Mohr da Silveira. Email: barbaramohrs@gmail.com 


\section{INTRODUÇÃO}

Os hábitos saudáveis permeiam as questões de saúde da população e, estão diretamente ligados com o fato do ser ou não saudável, além de estar associada ao surgimento de doenças crônicas. Como forma de manter hábitos saudáveis, cita-se o consumo de uma alimentação saudável e a prática regular de atividades físicas ${ }^{(1)}$.

Neste sentido, uma alimentação para ser classificada como saudável, além de ir ao encontro com os aspectos biológicos e socioculturais de cada indivíduo, deve estar associada ao uso sustentável do meio ambiente, ser consoante com as necessidades de cada fase da vida dos indivíduos, embasada na cultura alimentar, e, por fim deve ser acessivel na perspectiva financeira, harmônica em quantidade e qualidade(2).

O hábito do consumo de uma alimentação pode promover influência tanto na prevenção como também no desenvolvimento de doenças, neste sentido, a manutenção de uma vida saudável, por meio do consumo de alimentos saudáveis associada a prática regular de atividades físicas auxiliam na redução da prevalência das Doenças Crônicas não Transmissiveis (DCNT) na população $0^{(3-4-5)}$

Os hábitos saudáveis, alimentação e atividades físicas precisam ser estimuladas desde o nascimento e se manter até o final da vida. Nas crianças em particular pode ser reforçado no ambiente escolar, pois é um local onde as mesmas estão receptivas para novos aprendizados, podendo assim, as atividades influenciarem de modo positivo em seus estilos de vida assim como em suas famílias(b).

Diante disto, questiona-se: Quais as opiniões das familias sobre as atividades educativas desenvolvidas com as crianças na escola abordando hábitos saudáveis?

Este estudo teve como objetivo conhecer a opinião das familias sobre as atividades educativas desenvolvidas com as crianças na escola abordando hábitos saudáveis

\section{MÉTODO}

\section{Tipo de Estudo}

Estudo descritivo, exploratório, de abordagem qualitativa.

\section{Participantes da pesquisa}

Participaram das atividades lúdicas 51 crianças e suas respectivas familias que relataram suas opiniões sobre as atividades realizadas. Os critérios de inclusão foram: crianças na faixa etária entre seis a 9 anos. E os critérios de exclusão foram crianças ausentes durante três dias de atividades consecutivos.

\section{Local do estudo}

Escola da rede pública de educação em um município da grande Florianópolis - Santa Catarina

\section{Coleta dos dados}

Antes do início das atividades com as crianças na escola, foi realizado uma reunião com as famílias com o objetivo de esclarecimento sobre o projeto e o consentimento dos mesmos.

A partir do consentimento das famílias foram realizadas cinco atividades lúdicas, quinzenalmente, com as crianças que contemplavam aspectos de alimentação saudável e prática de atividades físicas.

A primeira atividade foi o jogo da memória. O objetivo desta era o reconhecimento dos alimentos, por meio de imagens de frutas e verduras impressas da internet. Para a segunda atividade foi construída uma Pirâmide dos Alimentos, como este assunto já havia sido tratado durante as aulas das crianças, definiu-se que seria uma boa forma das crianças relembrarem a temática. A terceira atividade intitulada "Recriando Pratos lúdicos", tinha como objetivo a reprodução de pratos temáticos utilizando frutas e verduras in natura para que as crianças manipulassem os alimentos. A quarta atividade denominada "Caixa Misteriosa", tinha como intuito a degustação de alimentos às cegas, a caixa utilizada na atividade possui uma abertura redonda que faz ligação com a sua parte posterior, transformando assim em uma canal de comunicação, assim, a parte frontal fica voltada às crianças, as quais não visualizam o que possui na parte posterior, que por sua vez. possui os alimentos devidamente higienizados e picados. Nessa atividade, os participantes com os olhos fechados, devem colocar as mãos através da abertura da caixa, pegar um alimento e através dos seus sentidos como olfato, tato e paladar tenta descobrir o que está degustando. Por fim, como quinta atividade foi prática de exercícios físicos chamada "Pega Bandeira". As turmas foram divididas em dois grupos, e após a divisão cada uma das equipes ganhou uma bandeira. Cada equipe tinha o objetivo de capturar a bandeira da equipe oposta ao mesmo tempo em que defendia a sua.

Após a realização das atividades, foi enviado às famílias, através das crianças, um questionário estruturado que continha questões fechadas e abertas, e essas abordavam as opiniões quanto às atividades que foram realizadas na escola com seus filhos sobre alimentação saudável e a prática de atividades físicas. $O$ período para a realização das atividades e coleta dos dados aconteceram nos meses de março a junho de 2018 .

\section{Procedimentos de análise dos dados}

Após a organização das respostas iniciou-se a análise dos dados que se deu por meio da proposta da Análise de Conteúdo, que tem como objetivo a categorização dos dados para melhor interpretação dos achados no estudo. A análise de conteúdos segundo Bardin pode ser compreendida em três 
fases: a pré-análise; exploração do material; e tratamento dos resultados ${ }^{(7)}$.

\section{Procedimentos éticos}

Foram respeitados todos os aspectos éticos descritos na resolução 466/2012 e com a aprovação do comitê de ética em pesquisa da Universidade Federal de Santa Catarina, através do Parecer: № 2.190.570. Todos os participantes assinaram o Termo de Consentimento Livre e Esclarecido em duas vias. Para preservar o anonimato dos participantes, identificou-se as opiniões das famílias com a letra " $F$ " seguido pelo algarismo arábico.

\section{RESULTADOS}

A partir da análise dos dados surgiram três categorias: Mudanças observadas nos hábitos cotidianos e nos discursos das crianças; O ensino dos hábitos saudáveis na escola; e Formas de abordar a temática na escola.

Mudanças observadas nos hábitos cotidianos e nos discursos das crianças

Os pais perceberam algumas mudanças tanto comportamentais quanto nas falas das crianças, pois os mesmos compartilhavam com suas familias as novidades que aprendiam durante as atividades na escola.

Alguns pais relataram que os filhos passaram a realizar mais atividades físicas, como por exemplo andar de bicicleta e até mesmo realizar atividades juntos aos pais, como demonstrado abaixo:

"Meu filho está comendo mais frutas e verduras, também está andando mais de bicicleta. " (F26)

"Ele faz os exercícios com a mãe exercício e faz caminhada. " (F29)

"[...] sobre atividades físicas também ficou mais disposta. $"($ F36)

"[...]comendo mais frutas e andando de bicicleta." (F42)

"Ele tem praticado mais exercícios comigo em casa, como pular corda, salto em distância." (F48)

Em outras falas, os pais comentaram sobre as crianças demonstrarem interesse em experimentar alimentos diferentes antes não provados, e ingerir mais frutas e verduras, como exemplificadas nos relatos abaixo:

"Ela passou a consumir mais frutas. Aumentou a sua aceitação em experimentar o que não conhece." (Fl)

"[...] têm 7 anos e nunca provou uma fruta e começou a aceitá-los desde então." (F3)

"Agora come algumas verduras que não comia antes." (F5)

"A experimentar frutas que antes não comia." (F7)

"[...] antes ela não conhecia o kiwi, agora ela gosta e pede pra comprar." (F15)

"Ele descobriu o gosto por outros alimentos que ainda era resistente à experimentar." (F50)

“[...] experimentou alimentos diferentes, sendo que ela é muito resistente a frutas e verduras." (F51)

\section{O ensino de hábitos saudáveis na escola}

Os pais trouxeram em suas opiniões sobre o ensino dos hábitos saudáveis no ambiente escolar. Surgiram falas que relacionam o ensino da saúde na escola e a sua importância para o desenvolvimento e manutenção da saúde das crianças.

“Excelente, pois está formando conceitos e padrões de comportamento que geram saúde e bem estar físico e emocional." (F4)

"Acho muito bom e importante para o futuro deles em questão de saúde. Com certeza no futuro irão se lembrar o que foi falado na escola." (F5)

"Acho bom porque os incentiva a se alimentar melhor e os incentiva também a praticar esportes." (F6)

"Para as crianças que não tem hábitos alimentares saudáveis, tudo o que for apresentado a elas de maneira interessante pode se transformar em curiosidade e a partir daí virar costume se alimentar bem e questionar o que faz mal à saúde." (F23)

"Isso é importante para a saúde da criança e seu próprio desenvolvimento." (F24)

"Eu acho muito importante ele já ter o conhecimento dentro da escola e saber que a alimentação saudável é muito importante. $\mathrm{E}$ as atividades físicas também, pois em casa tento mostrar as melhores maneiras pra ele." (F26)

"[...] porque assim eles aprendem que os alimentos saudáveis fazem bem para a saúde e que a atividade física também é bom." (F37)

"Acho de super importante o incentivo desde a infância, assim crescerão com bons hábitos saudáveis e serão prevenidos de problemas futuros, como a obesidade infantil que tem aumentado consideravelmente nos últimos anos." (F48)

Algumas familias trouxeram como relevante o fato de a criança estar no ambiente com os seus colegas, pois assim os estimula a experimentarem novos alimentos, como demonstradas abaixo:

"É muito importante, pois o fato de estar entre os amiguinhos, motiva mais a criança a aderir o que é proposto." (Fl)

"Bom, porque assim ela vê outras querendo e aceitando outros alimentos e também tem vontade de experimentar, o mesmo se diz as atividades esportivas." (F39)

“É muito interessante, principalmente na alimentação porque ele vendo outras crianças comendo ele também se interessa." (F46)

"Sou completamente a favor, pois ele vendo seus amigos provando facilitará para o seu consumo." (F3)

Outras famílias relataram a importância da continuidade 
do ensino da temática no ambiente escolar

"Se for de forma contínua (todo o ano letivo) acho que faria mais diferença sim, aprender mais sobre verduras, frutas e saladas e os benefícios delas, despertaria a curiosidade da criança em experimentar." (F19)

"É de muita importância, porém acho que deve ser um trabalho contínuo incluindo todas as séries." (F28)

"[...] eu como mãe acho muito bom e devia continuar, porque a [...] é muito alérgica e o médico pediu para ser mais saudável." (F40)

\section{Formas de abordar os hábitos saudáveis na escola}

Nesta categoria, os familiares opinam sobre as formas com que a temática poderia ser abordada na escola. Elencam como essencial o ensino da temática através de brincadeiras, atividades recreativas e conversas, ou seja atividades lúdica.

"Estimulando as crianças a comer o que nunca provaram, e oferecendo sempre uma fruta depois do lanche, como: banana, maçã ou laranja. Importante também oferecer frutas da época, como esse mês em nossa região encontra-se em abundância a bergamota e a laranja. " (F15)

"Incluindo aulas de culinária, promovendo lanche saudável, introduzir o dia da fruta, disponibilizar aulas de vôlei e futebol mais vezes na semana no contraturno." (F17)

"Ofertando alimentos saudáveis, oferecendo palestras aos pais e alunos e proporcionando atividades físicas que tenham a participação da família." (F23)

"Através de trabalhos e brincadeiras envolvendo as crianças, como oficinas feitas na cozinha, piqueniques nas turmas ou um dia por semana trabalhar essa alimentação." (F35)

"Na questão da alimentação, talvez poderiam ter aulas práticas com o contato da criança no alimento. Trazer mais modalidades de esportes para despertar algo que se identifiquem e talvez tenham o dom, mas por falta do conhecimento muitas vezes passa despercebido." (F48)

\section{DISCUSSÃO}

Tratar de temas voltados a saúde nas escolas, incluindo a alimentação saudável e a prática de atividades físicas, contribui para a reflexão das crianças sobre seus próprios hábitos e para a inserção de mudanças no seu cotidiano, para tanto, é de extrema importância que haja um incentivo a estas práticas na escola, principalmente se as intervenções forem voltadas ao lúdico, porém, para que serem efetivas, a temática deva ser abordada de forma transversal no currículo das escolas ${ }^{(8)}$

A realização de atividades lúdicas com crianças, é uma maneira de promover o aprendizado de uma forma facilitada e diferenciada, pois o aprender através de brincadeiras possibilita a aquisição de conhecimentos de forma mais descon- traída e dinâmica, ressalta-se que o lúdico chama a atenção dos alunos e os estimula a participar, instigando neles a curiosidade e o desejo de aprender mais sobre os conhecimentos que estão sendo repassados no ambiente escolar e em suas vidas $^{(9-10)}$

Percebeu-se que os resultados deste estudo apresentam semelhanças com o estudo que abordam os temas relacionados à saúde através de atividades lúdicas e realizadas de forma divertida possuem grande eficácia na divulgação de conhecimentos, principalmente sobre alimentação saudável e a prática de atividades físicas, além de auxiliar na adesão e manutenção dos bons hábitos devido ao maior envolvimento das crianças nas atividades ${ }^{(11)}$

As famílias do estudo demonstraram em suas opiniões preocupação com seus filhos e o desenvolvimento de doenças, como a obesidade. No Brasil, o número de casos de sobrepeso e a obesidade vêm crescendo, em todas as faixas etárias e em ambos os sexos, em todos os níveis de renda ${ }^{(12)}$.

Esse aumento de casos está relacionado aos elevados indices de inatividade físicas, aliado a uma alimentação rica em alimentos gordurosos, as quais possuem alta densidade energética, porém baixa concentração de nutrientes, possuem relação direta com os valores no aumento da obesidade e outras doenças crônicas, como diabetes, hipertensão e outras doenças cardiovasculares ${ }^{(2-13)}$.

Como fator significativo para o aumento dos índices de obesidade tem-se o alto consumo dos alimentos industrializados e multiprocessados, que possuem em sua composição grande quantidades de açúcares, gorduras e outros ingredientes prejudiciais à saúde, associado à inatividade física, e o estilo de vida das famílias, que por falta de tempo para realizarem refeições completas, e saudáveis aumentam o consumo de alimentos industrializados que são mais rápido e que facilita o dia a dia ${ }^{(14-15)}$

Outra questão que pode contribuir para o aumento do consumo de alimentos industrializados, é a veiculação midiática, onde o marketing alimentar é maciço, as empresas buscam vender cada vez mais produtos e, para tanto, fazem uso do lúdico, utilizando bonecos e outros personagens infantis, tendo assim influência direta nos hábitos alimentares principalmente das crianças, as quais acabam tendo uma alimentação rica em açúcares e gorduras ${ }^{(16)}$.

Mais especificamente sobre a alimentação de escolares, pode-se observar que a alimentação desta faixa etária possui níveis significantes de açúcar e sódio, o que pode gerar determinados impactos e repercussões para suas vidas adultas ${ }^{(17)}$.

Os hábitos que os indivíduos levam para suas vidas adultas, em sua maioria são criados na infância, por isso, o costume e o conhecimento acerca da importância de manter uma alimentação saudável principalmente no combate às doenças 
crônicas deve estar presente na vida cotidiana desde a primeira infância(18).

O ensino e o aconselhamento sobre as relações da prática de exercícios físicos e hábito de alimentação saudável, tem como maior objetivo de levar o conhecimento para promoção da saúde da população em geral, e principalmente, em crianças (19). E neste aspecto o contexto familiar, pode promover a interação das crianças com os alimentos de forma mais saudável possivel ${ }^{(20)}$.

Portanto, é necessário a inclusão das famílias nas atividades escolares, na construção do aprendizado, e na consequente formação de hábitos das crianças. Sendo que estas podem estar associadas ao uso de jogos, associado ao Política de Saúde na Escola, sendo considerada uma ferramenta importante para as equipes de atenção primária, assim como para os profissionais da educação, uma vez que proporcionam ações sustentáveis se desenvolvidas e compartilhadas de maneira igual com pais, educadores e profissionais de saú$\mathrm{de}^{(21)}$.

Em um estudo que buscava a opinião dos pais sobre as atividades na escola de ensino fundamental e médio, demonstrou que a forma lúdica envolvendo o brincar, refletir e aprender, despertam nas famílias o desejo de outras atividades de educação em saúde(22).

Para tanto, o ambiente escolar é considerado um lugar propício para a disseminação de informações e também para o estímulo de práticas saudáveis, contribuindo assim para a promoção da saúde em longo prazo, diminuindo as chances do desenvolvimento da obesidade tanto na infância quanto da vida adulta(11).

Assim, a escola, as crianças e suas famílias podem ser estimuladas a desenvolver hábitos de vida saudáveis e que poderão perdurar para a vida adulta.

\section{Limitações do estudo}

Acredita-se que as limitações deste estudo foram a quantidade de atividades realizadas com as crianças, pois sabe-se que não foram suficientes para promover mudanças mais visiveis e, por isso recomenda-se mais estudos sobre essa temática. Para tanto, indica-se a realização de novos estudos nesta temática com metodologias semelhantes, para que seja possivel observar maiores mudanças nos hábitos das crianças.

\section{Contribuições do estudo para a prática de enfermagem}

A contribuição está na riqueza de cuidados que podem ser desenvolvidos no ambiente escolar, principalmente quando se pensa em duas vertentes: no papel do enfermeiro como educador e na promoção da saúde das crianças no ambiente escolar.

\section{CONSIDERAÇÕES FINAIS}

Através deste estudo foi possivel conhecer as opiniões das famílias em relação às atividades que foram realizadas com as crianças sobre alimentação saudável e prática de atividades físicas, assim como outras preocupações relacionadas a saúde das crianças.

Pode-se observar que as familias perceberam mudanças, por mais que fossem sutis, nos hábitos alimentares das crianças, onde relataram que seus filhos, após as atividades realizadas, demonstraram maior interesse em experimentar novos alimentos e também aumentaram o seu consumo de frutas e verduras, assim como passaram a praticar mais atividades físicas.

Algumas famílias reforçaram a importância do ensino da temática na escola para a promoção da saúde das crianças, com isso, pode-se concluir que os mesmos percebem o quão é importante a manutenção de bons hábitos nas crianças para a prevenção de doenças na vida adulta. Desta forma, as atividades lúdicas são efetivas para o incentivo de mudanças nos hábitos cotidianos das crianças.

Para tanto, indica-se a realização de novos estudos nesta temática com metodologias semelhantes, para que seja possível observar maiores mudanças nos hábitos das crianças.

\section{Contribuição dos autores}

Bárbara Mohr da Silveira: Concepção e desenho, análise e interpretação dos dados, redação do artigo, revisão crítica, revisão final;Luciara Fabiane Sebold: Concepção e desenho, análise e interpretação dos dados, redação do artigo, revisão crítica, revisão final;Larissa Evangelista Ferreira: Análise e interpretação dos dados, redação do artigo, revisão crítica, revisão final;

Juliana Balbinot Reis Girondi: Redação do artigo, revisão crítica, revisão final;Lúcia Nazareth Amante: Redação do artigo, revisão crítica, revisão final:Juliana Simas Justino: Redação do artigo, revisão crítica, revisão final. 


\section{REFERÊNCIAS}

1. Toledo MTT, Abreu MN, Lopesi ACS. Adesão a modos saudáveis de vida mediante aconselhamento por profissionais de saúde. Rev Saúde Pública [internet]. 2013 [cited 2019 Fev 19]:47(3):540-8. Available from: http://www.scielo.br/scielo.php?pi$d=S 0034-891020130003005408$ script=sci_abstract\&tlng=pt

2. Ministério da Saúde (BR), Secretaria de Vigilância à Saúde, Secretaria de Atenção à Saúde. Política Nacional de Alimentação e Nutrição. Brasilia: Ministério da Saúde, 2013.

3. Souza EB. Transição nutricional no Brasil: análise dos principais fatores. Cadernos Unifoa [Internet]. 2010 [cited 2018 Ago 17]:3:49-53 Available from: http://revistas.unifoa.edu.br/index.php/cadernos/ article/view/1025

4. Bezerra IN, Cavalcante JB, Moreira TMV, Mota CC, Sicheiri R. Alimentação fora de casa e excesso de peso: uma análise dos mecanismos explicativos. Rev Bras Promoç Saúd [Internet]. 2016 [cited 2018 Ago 17]:29(3):455-461. Available from: https://periodicos.unifor.br/ RBPS/article/view/5240

5. Silva ADC, Castro AGO, Pereira APN, Souza AAR, Amorim PRA, Reis RC. Hábitos alimentares e sedentarismo em crianças e adolescentes com obesidade na admissão do programa de obesidade do hospital universitário bettina ferro de souza. Rbone [Internet]. 2017 [cited 2018 Ago 17];11(61):39-46. Available from: http://www.rbone.com.br/index. php/rbone/article/view/486

6. Bernardi AP, Maciel MA, Baratto I. Educação nutricional e alimentação saudável para alunos da Universidade Aberta a Terceira Idade (UNATI). Rbone [Internet]. 2017 [cited 2018 Ago 17];11(64):224-231.

Available from: http://www.rbone.com.br/index.php/rbone/article/ view/523

7. Bardin L. Análise de Conteúdo. 5 ed. São Paulo: Almedina; 2011. 8. Silva FMA, Smith-Menezes A, Duarte MFS. Consumo de frutas e vegetais associado a outros comportamentos de risco em adolescentes no Nordeste do Brasil. Rev Paul Pedatr [Internet]. 2016 [cited 2018 Ago 18]:34(3):309-315. Available from: http://www.scielo.br/pdf/rpp/ v34n3/pt_0103-0582-rpp-34-03-0309.pdf

9. Kaufmann-Sacchetto K, Madaschi V, Barbosa GHL, Silva PL, Silva RCT, Filipe BTC, Souza-Silva JR. O ambiente lúdico como fator motivacional na aprendizagem escolar. Cadernos de Pós-graduação em Distúrbios do Desenvolvimento [Internet]. 2011 [cited 2018 Ago 18]:11(1):28-36. Available from: http://editorarevistas.mackenzie.br/ index.php/cpgdd/article/view/11170/6934

10. Ferreira MLS. A brincadeira e o aprender: um olhar psicopedagógico do processo de aprendizagem infantil [Trabalho de Conclusão de Curso]. Curso de Pedagogia: Universidade Federal da Pará, 2016.

11. Lopes MMD, Della BL. Educação nutricional e práticas alimentares saudáveis na infância. REINPEC [Internet]. 2017 [cited 2018 Ago 18];3(1):200-210. Available from: http://reinpec.srvroot.com:8686/ reinpec/index.php/reinpec/article/view/264

12. Instituto Brasileiro de Geografia e Estatística [Internet]. Pesquisa de Orçamentos Familiares 2008- 2009: antropometria e estado nutricional de crianças, adolescentes e adultos do Brasil - [cited 2018 Ago 17]. Available from: https://biblioteca.ibge.gov.br/visualizacao/livros/ liv45419.pdf
13. Silva JG. Teixeira MLO, Ferreira MA. Eating during adolescence and its relations with adolescent health. Text Context Nursing [Internet]. 2014 [cited 2018 Ago 18];23(4): 1095-1103. Available from: http://www. scielo.br/pdf/tce/v23n4/0104-0707-tce-23-04-01095.pdf

14. Louzada MLC, Martins APB, Cannela DS, Baraldi LG, Levy RB, Claro RM, Moubarac J, Cannon G, Monteiro CA. Alimentos ultraprocessados e perfil nutricional da dieta no Brasil. Rev. Saúde Pública [Internet]. 2015 [cited 2018 Ago 18]:49(38):1-11. Available from: http://www.scielo. br/scielo.php?pid=s0034-89102015000100227\&script=sci_arttext\&tlng=pt

15. Claro RM, Santos MAS, Oliveira TP, Pereira CA, Szwarcwald CL, Malta DC. Consumo de alimentos não saudáveis relacionados a doenças crônicas não transmissiveis no Brasil: Pesquisa Nacional de Saúde, 2013. Epidemiol. Serv. Saúd [Internet]. 2015 [cited 2018 Ago 18];24(2):257-265. Available from: http://www.scielo.br/scielo.php?pid=S2237-96222015000200257\&script=sci_abstract\&tlng=pt

16. Santana MKL, Oliveira CM, Clemente HA. Influência da publicidade de alimentos direcionada ao público infantil na formação de hábitos alimentares. Revista Uni-rn [Interet]. 2015 [cited 2018 Ago 18];14(1/2):125-136. Available from: http://www.revistas.unirn.edu.br/ index.php/revistaunirn/article/view/355/300

17. Fisberg M, Previdelli AN, Del'Arco APWT, Tosatti A, Nogueira-de-Almeid CA. Hábito alimentar nos lanches intermediários de crianças escolares brasileiras de 7 a 11 anos: estudo em amostra nacional representativa. International Journal Of Nutrology [Internet]. 2016 [cited 2018 Ago 18];9(4):225-236. Available from: http://nutrociencia. com.br/wp-content/uploads/2017/01/2016-NutriBrasil-7-a-11-anos-1. pdf

18. Silveira MGG. Prevenção da obesidade e de doenças do adulto na infância. I V. Petrópolis: Vozes, 2017.

19. Lopes ACS, Toledo MTT, Câmara AMCS, Menzel HK, Santos LC. Condições de saúde e aconselhamento sobre alimentação e atividade física na Atenção Primária à Saúde de Belo Horizonte-MG. Epidemiol. Serv. Saúd [Internet]. 2014 [cited 2018 Ago 18];23(3):475-486. Available from: http://www.scielo.br/scielo.php?pi$d=S 2237-962220140003004758$ script=sci_abstract\&tlng=pt

20. Silva GAP, Costa KAO, Giugliani ERJ. Infant feeding: beyond the nutritional aspects. J Pediatr [Internet]. 2016 [cited 2017 Ago 17]:92(3):52-57. Available from: http://www.scielo.br/scielo.php?scrip$\mathrm{t}=\mathrm{sci}$ arttextEpid=S0021-75572016000400002\#aff1

21. Vasconcelos CMR, Vasconcelos EMR, Vasconcelos MGL, Azevedo SB, Raposo MCF, Vasconcelos ALR. Validation of an educational tool on healthy eating for schoolchildren. Rev. Nutr [Internet]. 2018 [cited 2019 Fev 18];31(5):479-488. Available from: http://www.scielo.br/scielo.php?script=sci_arttext\&pid=S1415-52732018000500479\&lng=pt. http://dx.doi.org/10.1590/1678-98652018000500005.

22. Souza TCF, Carvalho JN. A percepção de pais sobre projeto de extensão universitária em escola amazônica. Enferm. Foco [Internet]. 2018 [cited 2018 Fev 18];9(3):25-29. Available from: http://revista. cofen.gov.br/index.php/enfermagem/article/view/1148 